\title{
MAESTRAS RURALES CRUCEÑAS, DEPARTAMENTO DE NARIÑO - COLOMBIA, VOCACIÓN, CONVICCIÓN Y PRINCIPIOS: IRMA CECILIA PALACIOS ARCOS, IDA VICTORIA BOLAÑOS CERÓN
}

\author{
José Oliden Muñoz Bravo1 \\ Grupo de investigación GIDEP \\ nsmlacruz@gmail.com
}

\begin{abstract}
Resumen
El artículo trata sobre la práctica de las maestras rurales en Colombia durante el periodo comprendido entre 1946 a 1994, esta investigación toma una descripción general como estaba articulado el trabajo de las normales y cuáles fueron sus fundamentos en la formación, capacitación, orientación y perfeccionamiento de las nuevas maestras. El estudio Hermenéutico toma la experiencia de dos maestras cruceñas, quienes entregan toda una vida al servicio de la enseñanza y aplicación en valores de los niños de las comunidades donde ejercieron su profesión. La formación de maestras a nivel de las normales permitió desenvolverse en los contextos socioculturales donde se articula el contacto directo con la vida campesina, desarrollando un mutuo entendimiento que conduciría a llegar a las familias y compartir múltiples oficios que se impartían en las tardes y en las noches.

Analiza la vida de dos maestras rurales, quien a través de la formación y dedicación entregan su vida a la educación primaria en Colombia durante el siglo XX desde una perspectiva de 35 años de servicio y compromiso con los niños de la comunidad del municipio de la Cruz Nariño. Se estudian los cambios institucionales y organizacionales que ocurrieron frente a su responsabilidad en la educación.
\end{abstract}

Palabras clave: Maestras rurales, educación, orientación, vocación, convicción.

1 Doctor en Ciencias de la Educación (RUDECOLOMBIA Universidad de Nariño), Magister en educación de Adultos (Universidad San Buenaventura - Universidad de Mariana), Especialista en Educación: Administración Educativa (Universidad de Nariño), Licenciado en Educación Primaria (Universidad San Buenaventura - Universidad Mariana), Profesor titular Escuela Normal Superior del Mayo (La Cruz, Nariño). 


\title{
RURAL CRUCEÑA PROFESSORS OF THE DEPARTMENT OF NARIÑO, COLOMBIA- VOCATION, CONVICTION, AND PRINCIPLES: IRMA CECILIA PALACIOS ARCOS, IDA VICTORIA BOLAÑOS CERÓN
}

\begin{abstract}
This article discusses the practice of rural teachers in Colombia during the period of 1946 to 1994. The investigation is comprised of a general description of the work of the Normal schools and its foundations in formation, training, guidance, and improvement of new teachers. This hermeneutical study takes the experience of two teachers from Santa Cruz who dedicated a lifetime to the service of teaching and application of values, to the children of the communities where they practiced their profession. The training of these Normal school teachers unfolded in socio-cultural contexts where direct contact with peasant life is articulated, and a mutual understanding was developed that would lead to bonding with families and the sharing of multiple trades that were taught in the evenings and nights.

This paper analyzes the life of two rural teachers, who through their training and dedication, dedicated their lives to primary education in Colombia during the 20th century, from a perspective of 35 years of service and commitment to the children of the community of La Cruz, Nariño. It focuses on the institutional and organizational changes that occurred as a result of their work in education.
\end{abstract}

Keywords: Rural teachers, education, orientation, vocation, conviction.

\section{MESTRES RURAIS CRUCEÑAS, DEPARTAMENTO DE NARIÑO - COLÔMBIA, VOCAÇÃO, CONVICÇÃO E PRINCÍPIOS: IRMA CECILIA PALACIOS ARCOS, IDA VICTORIA BOLAÑOS CERÓN}

\begin{abstract}
Resumo
0 artigo aborda a prática de professores rurais na Colômbia durante o período de 1946-1994, esta pesquisa tem uma visão geral como foi articulado o trabalho das normais e quais foram seus fundamentos em educação, treinamento, orientação e desenvolvimento de as novas professoras. 0 estudo hermenêutica leva a experiência de duas professoras Cruceñas, que entregam uma vida ao serviço do ensino e aplicação valores das crianças nas comunidades onde exerciam sua profissão. Formação de professoras ao nível das normais permitiu o desenvolvimento nos contextos sociais e culturais onde o contato direto com a vida rural é articulada, o desenvolvimento de um entendimento mútuo de que levaria alcançar famílias e compartilhar vários ofícios que foram realizados nas tardes e noites.

Examina a vida de duas professoras rurais, que por meio de treinamento e dedicação dão suas vidas ao ensino primário na Colômbia durante o século XX a partir da perspectiva de 35 anos de serviço e compromisso com as crianças da comunidade no município de La Cruz Nariño. Se estudam As mudanças institucionais e organizacionais que ocorreram em face de sua responsabilidade na educação.
\end{abstract}

Palavras-chave: Professorass rurais, educação, orientação, vocação, convicção. 


\section{Introducción}

Las Escuelas Normales Rurales para mujeres fueron creadas por la ley 12 del 17 de diciembre de 1934, tenían como finalidad especifica preparar las maestras para la educación rural primaria. La formación de las maestras rurales estaba dada en dos años de internado, las cuales se fundamentaban en conocimientos elementales, trabajos manuales y agrícolas e higiene ${ }^{2}$.

Era necesario, entonces, la alfabetización del proletariado agrícola, de ahí el énfasis del liberalismo en la educación rural con la creación de normales rurales en 1934 por la Ley del 17 de diciembre y la enseñanza vocacional agrícola para buscar la tecnificación de la educación rural y de la producción agrícola ${ }^{3}$, en esta lógica se insiste, en el periodo, en la programación de la educación aldeana por medio del radio, cine y bibliotecas populares ${ }^{4}$.

Los programas para las Normales Rurales, excluían, por primera vez en el siglo la signatura de pedagogía y se dirigían a una educación centrada en los problemas y necesidades de la vida rural5. Para López de Mesa, las maestras formadas debían convertirse en dirigentes del campesino, encauzando sus esfuerzos a la salvación de la raza por medio de la higiene, el desarrollo de la economía y la agricultura, la moralización de la población y el desarrollo de su sentido estético ${ }^{6}$. Dentro del propósito de modernización de la cultura, para López de Mesa era fundamental la transformación de la estética de las maestras rurales. Se les dotaba de becas para el vestido con el propósito de que aprendieran a seleccionar y confeccionar sus ropas de acuerdo a su estatura y color, así como para que completaran el ajuar de una mujer moderna, el cual incluía sombreros, calzados, ropa interior, vestido de baño y de gimnasia, así como el atuendo para excursiones de diversos climas. Este cambio según López de Mesa traería efectos pedagógicos ya que "Una maestra de atractivo personal será el mejor ejemplo y obtendrán mejores triunfos disciplinarios que no cualquier esperpento antipático al ojo inquisitivo de los educandos"7.

La educación Normalista, según el decreto 71 de 1939, estableció el nuevo pensum único nacional para que el Ministerio aprobara los certificados de estudio de sus graduandos ${ }^{8}$.

En armonía con los nuevos rumbos de la política educativa estatal, el nuevo sistema de formación de los futuros maestros se basaba en la creación de sus

2. Aline Helg, La educación en Colombia: 1918-1957 (Bogotá: Universidad Pedagógica y Tecnológica de Colombia, Plaza y Janes, 1987), 74

3. Fernán González, "Educación y estado en la historia de Colombia", Revista Colombiana de Educación, No. 4 (1979): 81.

4. González, "Educación y estado en la historia de Colombia", 90.

5. Luis López de Mesa, "Tres instituciones pedagógicas Nacionales”, Revista de Indias. Vol. 1, No. 7 (1937): 54.

6. Ministerio de Educación Nacional, Memoria de 1935, 31.

7. López de Mesa, “Tres instituciones pedagógicas Nacionales”, 54.

8. Ministerio de Educación Nacional, Programas de Estudio para las Escuelas Normales Regulares de la República, 1939. 
valores y una pedagogía de fundamento social y político. Los estudiantes debían compenetrarse con la misión social de la escuela.

En 1935, la enseñanza primaria oficial contaba 10.604 maestros. Globalmente el magisterio era en su $30 \%$ graduados, pero la desigualdad entre el campo y las zonas urbanas era fuerte: casi la mitad de los maestros urbanos era normalistas graduados, mientras que solo el $6 \%$ del personal docente rural tenía grado. Es decir que prácticamente la totalidad del magisterio en el campo no tenía ninguna formación pedagógica. Además, de diez maestros, siete eran mujeres solteras; en el campo, el $90 \%$ de los institutores eran de sexo femenino, en las ciudades solamente el $55 \%{ }^{9}$. La no calificación del magisterio colombiano y el peso de trabajo femenino tenían su origen en el nivel de sueldos y el estatuto social de la profesión, tradicionalmente muy bajos en el país, que generaban un círculo vicioso de condiciones socio-económicas inferiores y subcalificación. Así el carácter femenino de la profesión influía también en la consideración social que hombres y sociedad en general tenían de la tarea de educador.

El sueldo de un maestro rural era apenas la mitad del recibido por un maestro en la ciudad. No existía un escalafón nacional de salarios relacionado con la formación y los años de servicio. Además, el cumplimiento en el pago de los sueldos dependía del estado fiscal de los departamentos.

La Ley 2a. de 1937 fijó un salario mínimo para los maestros de primaria equivalente a 40 pesos mensuales. Creó el escalafón nacional del magisterio y estableció que podrían ser nombrados maestros de escuela solamente los que llenaban los requisitos fijados en éste. Para su clasificación, impuso un examen de conocimientos a todo el magisterio en ejercicio que provocó una fuerte reacción de los maestros, especialmente los recién salidos de los institutos normales.

En Colombia a fines de 1934 se promulgó la ley 12 creando las escuelas normales rurales. En ellas se daba a niñas con estudios completos de primaria una formación pedagógica de dos años abarcando conocimientos literarios, actividades del campo y desarrollo de la personalidad. En 1938 funcionaban cuatro normales, rurales, en Bogotá, Gigante (Huila), Santa Marta y Popayán con un total de 330 alumnas. A pesar de que muchas de las maestras graduadas en esas escuelas no volvieron al campo, fue una medida que permitió la formación corta del personal docente. En el mismo orden de ideas se crearon en las tres normales nacionales y en varios departamentos cursos de vacaciones para maestros graduados o sin grado, en los cuales recibían una orientación pedagógica hacia los métodos modernos. El Ministerio de Educación decidió también la creación de un servicio de maestros ambulantes, cuyo programa era muy ambicioso. En 1936 debían salir los cien primeros maestros del servicio no para alfabetizar o enseñar, sino para explicar la economía, el comercio, la geografía colombiana a un campesinado en su gran mayoría analfabeta. La segunda parte del proyecto preveía la enseñanza por

9. MEN., Memoria de 1935, 250-251. 
radio ${ }^{10}$. Este ambicioso programa no se realizó, como tampoco se había logrado implantar el servicio voluntario femenino.

Durante el periodo comprendido entre 1940 y 1949 se establecieron ocho más. Según datos de Betancourt Mejía11, para el año 1956 estaban en funcionamiento dieciocho escuelas normales rurales nacionales, nueve departamentales y dieciséis privadas. De las dieciocho nacionales, solamente eran dos de varones y, de las veinticinco departamentales y privadas, veinte eran de mujeres y cinco de hombres ${ }^{12}$.

Este artículo analiza la vida de dos maestras rurales, quien a través de la formación y dedicación entregan su vida a la educación primaria en Colombia durante el siglo XX desde una perspectiva de 35 años de servicio y compromiso con los niños de la comunidad del municipio de la Cruz Nariño. Se estudian los cambios institucionales y organizacionales que ocurrieron. Se analiza la financiación y el pago de salarios frente a su responsabilidad en la educación.

En Colombia, las Escuelas Normales Femeninas se organizaron durante el gobierno liberal radical, la formación de maestras correspondió al interés de los liberales en la educación, la educación femenina fue una gran preocupación de los gobiernos radicales en Colombia con el fin de actualizar la educación fundamentada en valores la cual podía irradiar desde las familias a la escuela.

A finales del siglo XIX, con el decreto 356 del 27 de agosto de 1874, Santiago Pérez, presidente de los Estados Unidos de Colombia, reglamentó lo correspondiente a las Escuelas Normales Femeninas. Se determinó establecer una Escuela Normal de institutoras en cada uno de los Estados donde no exista ningún plantel de la misma clase, de conformidad con el mismo decreto ${ }^{\mathbf{1 3}}$.

Entre las reglamentaciones más importantes sobre la educación primaria se encuentra la descentralización administrativa del sistema escolar, por medio de la cual se les encargó a los departamentos la financiación, el pago de maestros, la dirección, la protección y la supervisión de la educación primaria. A los municipios se les ordenó suministrar los locales y enseres para el funcionamiento de las escuelas urbanas y rurales, siendo los Consejos municipales los encargados de apropiar los recursos necesarios para suplir dichos requerimientos; y a la Nación se le asignó la función de inspeccionar y proporcionar los útiles y textos a todas las escuelas primarias del país. En general, la educación primaria debería estar

10. El Tiempo, 22 de noviembre de 1935. Memoria de 1938 (cuadro en anexo) señala sin embargo, 14 maestros y 11 maestras ambulantes este año (1935), primera edición. Número 1531.

11. Gabriel Betancourt Mejía (Compilador), Documentos para la historia del planteamiento integral de la educación. Informe del proyecto para el Primer Plan Quinquenal de Educación, Vol. II (Bogotá: Universidad Pedagógica Nacional, 1984), 72.

12. Alba Nidia Triana Ramírez, "Escuelas Normal, Rural, Agropecuaria y de Campesinas en Colombia: 1934-1974", Revista Historia de la Educación en Colombia, Vol. 13, No. 13 (2010): 205.

13. Decreto 356 de 27 de agosto de 1874. Art. 1. Presidente Santiago Pérez, Escuelas Normales de mujeres. Estados Unidos de Colombia. 
orientada a preparar a los alumnos para la agricultura, la industria fabril y el comercio.

Las escuelas primarias se dividieron en urbanas, rurales y nocturnas, cada una de ellas era regida por diferentes normas. Por ejemplo, la escuela urbana constaba de seis años de enseñanza, los dos primeros llamados elementales, los dos siguientes escuela media y los últimos años escuela superior, mientras que la escuela rural solo constaba de tres años de instrucción primaria y era alternada según género.

En la formación de las maestras rurales era de vital importancia la enseñanza de la religión católica y la moral, las mujeres eran imagen moral de su familia e imagen de la escuela y como maestra debía ser modelo de conducta frente a su preocupación permanente por la formación y el comportamiento moral de las estudiantes maestras.

Sáenz expresa al respecto:

El pensum académico de las normales rurales fue reformado en 1945, a este se agregó un año de estudio, con énfasis en actividades relacionadas para la vida rural. "En matemáticas se introdujeron una serie de elementos de administración y manejo financiero para la tecnificación y modernización de la producción agrícola, como la contabilidad agrícola, la cual incluía el manejo de campañas comerciales y cooperativas agrícolas, el uso de libro de pérdidas y ganancias, la elaboración de documentos comerciales y de presupuestos domésticos, escolares, municipales, departamentales y nacionales. Igualmente, se incluyeron temas relacionados con el crédito agrario y ganadero, y con la elaboración de inventario, avalúos y libros de jornales ${ }^{14}$.

Los sueldos que recibían los maestros eran muy bajos relativamente. Mientras un profesor en la zona urbana ganaba a comienzo de los años treinta \$45 mensuales, un empleado de los ferrocarriles ganaba en promedio $\$ 82 .{ }^{15}$ De otra parte, los sueldos de los maestros se vieron gravemente afectados por la recesión económica. De acuerdo con las Memorias del Ministerio de Educación Nacional de 1931, los sueldos de los maestros se redujeron significativamente, llegando a "sueldos de emergencia” como él los llamó. El problema era aún más grave debido a que estos sueldos no se pagaban con puntualidad, perjudicando considerablemente a los maestros. Como no existía un escalafón nacional, con anterioridad a 1937, las diferencias salariales entre las regiones eran preocupantes, mientras que en 1936 un maestro en Antioquia, Caldas o Cundinamarca podía ganar \$60 mensuales, en Nariño solamente se les podía pagar la mitad, de hecho habían maestras de escuela rurales que recibían escasamente $\$ 18$ mensuales.

14. Javier Sáenz, Oscar Saldarriaga, y Armando Ospina, Mirar la infancia: pedagogía, moral y modernidad en Colombia, 1903-1946 (Medellín: Editorial Universidad de Antioquia, 1997), 369.

15. M. T, Ramírez, y Á. Pachón, La infraestructura de transporte en Colombia durante el siglo XIX (Bogotá: Banco de la República y Fondo de Cultura Económica, 2006). 
En 1945, por medio del Decreto 1790, se modificaron una vez más los planes de estudio de las normales regulares ${ }^{16}$. Los nuevos programas constituyen un paso más en ese proceso social, cultural y político del país.

El proceso de transformación de la formación de maestros, los cambios en el saber y la práctica pedagógica durante este periodo tienen una temporalidad y unas causas que le dieron una autonomía ante los cambios políticos. En 1933 se unificó a nivel nacional la enseñanza normalista, en 1934 se crearon las Normales Rurales y en 1939 y 1945 se modificaron una vez más los planes de estudio ${ }^{17}$. A pesar de la multitud de normas programáticas e institucionales como la disminución de la intensidad horaria y la reducción de la carga académica por el debilitamiento fisiológico y psicológico de los futuros maestros.

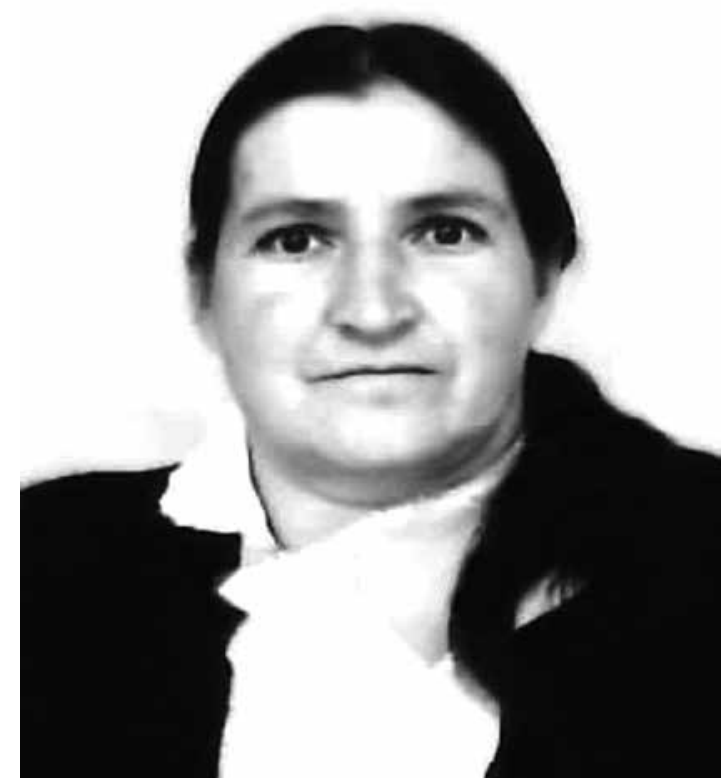

Fuente. Irma Cecilia Palacios Arcos

Irma Cecilia Palacios Arcos, maestra rural de 80 años, nacida en la Cruz Nariño Comenta:

Nacida en la Cruz Nariño, 11 de enero de 1936, hija de Alejandro Palacios Realpe y Ermelina Arcos Delgado, mis hermanos: Soledad, Analina, Eduardo Roque,

16. Ministerio de Educación Nacional, Plan de estudios para las Escuelas Normales Regulares (Bogotá: Imprenta Nacional, 1946).

17. República de Colombia, Memoria del Ministerio de Educación al congreso de 1946 (Bogotá: Imprenta Nacional, 1946), 13. 
Zoila Eva, María Jesús, Alejandrina y León, fuimos muy bien formados con la rectitud de mis padres donde siempre perduraba el respeto, la responsabilidad, la confraternidad y la armonía familiar, mi inclinación por la educación nace desde el seno familiar donde nos inculcaban y enseñaban muchas cositas que fueron alimentando esa llamita de ser maestra y servir una comunidad. Somos cuatro hermanos maestros en mi familia que se ha dedicado toda una vida en formar y educar a los niños y jóvenes para bien de las futuras generaciones.

"Hace un análisis de la educación de aquella época, la Normal era de cuatro años y bastante dura y fuerte nos exigían al máximo y salía con el título de Maestra Rural y recibimos las materias de matemáticas, Física, química, naturales, sociales, español, educación física, practica pedagógica para preparar el derrotero de las clases la forma en que se debe dictar la clase, la forma de tratar a los alumnos, teníamos que dar clases de lunes a sábado y para graduarse era el mes completo de la práctica. Fui graduada en 1947 y en nuestro grupo fuimos todas mujeres y la rectora era Nohemí Salcedo y en la anexa la directora era la profesora Ernestina Bolaños y la Normal se llamaba Normal Rural de Señoritas.

Las mujeres recibían culinaria, tejidos, costura, máquinas para coser diferentes vestidos. Al momento de graduarnos nos entregaban el nombramiento y me enviaron a Peña Negra queda muy cerca de Belén y luego pase a San Gerardo, Tajumbina, la Estancia y en Juan López donde ya me jubile. La educación era muy estricta sobretodo en la moral, ya que fuimos educadas con monjas, con respeto, responsabilidad y cumplimiento.

El mensaje como maestra educar a los niños es una labor muy dura pero grata"

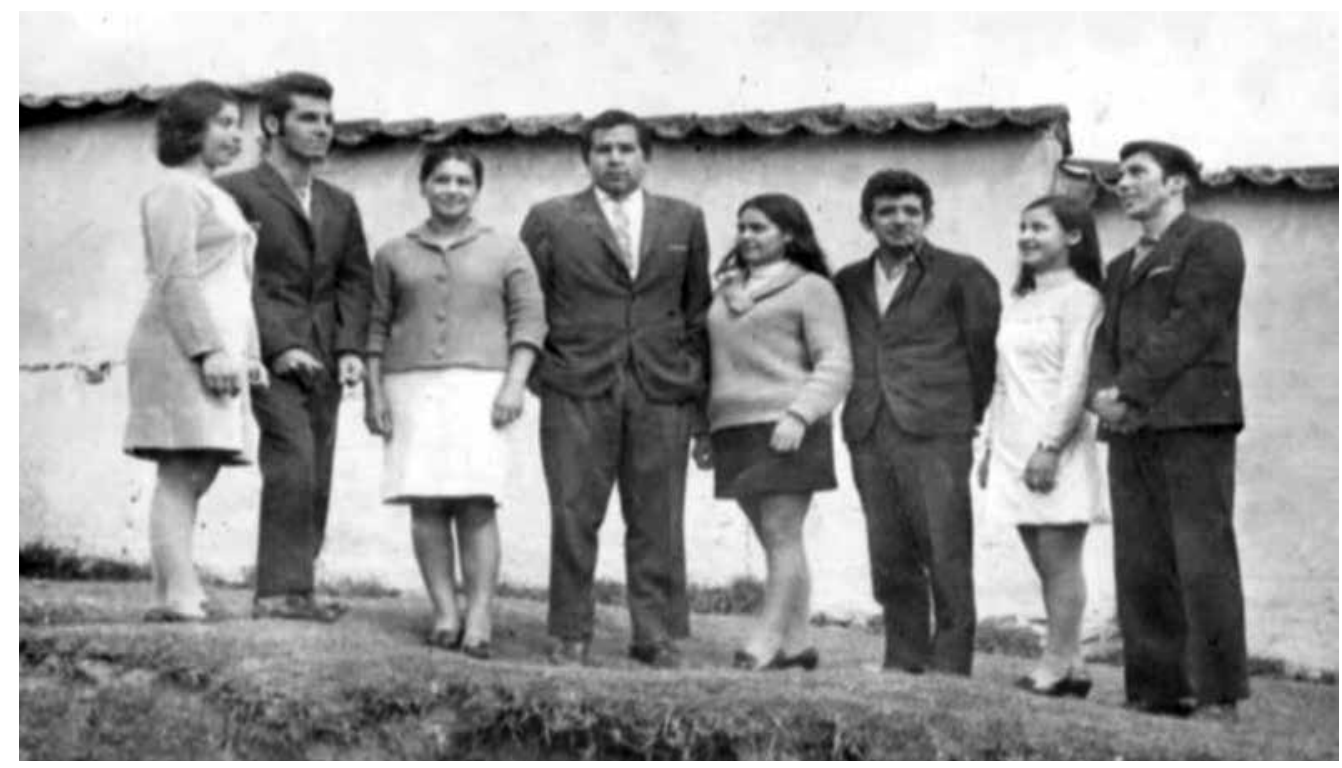

Fuente. Ida Victoria Bolaños, 1960. 
Ida Victoria Bolaños Cerón. Maestra Rural de 81 años, nacida en Belén Nariño, y su formación, profesión y vida familiar la desarrolla en el municipio de La Cruz Nariño. Comenta:

Mi patria chica es Belén (Nariño), nací del matrimonio de Laureano Bolaños y Romelia Cerón Gómez, mi padre fue agricultor, comerciante, ganadero y en La Cruz fue secretario de la alcaldía. Mi madre estudió en el municipio de San Pablo en el colegio de las madres Bethlemitas, fue profesora en Belén, después de casada renunció porque no era permitido que una profesora siguiera su docencia como lo hacen ahora, tenían que dedicarse completamente a su hogar.

En mi casa fuimos nueve hermanos (Luis, Arnulfo, Hernán, Alberto, Armando, Eduardo Alen, Carlos Humberto, Silvio Arturo y Ida Victoria) de los cuales estudiamos primaria y secundaria siete, trabajaron como profesores tres en Neiva (Huila), una en La Cruz, otro en Pensilvania Caldas, dos de ellos terminada la Normal Superior del Mayo de La Cruz, continuaron sus estudios en la Universidad de Tunja y otro en Medellín, luego se fueron a especializar al Brasil y a Francia, el hermano que fue al Brasil es traumatólogo y trabaja en Pasto y el que fue a Francia fue profesor de la Universidad de Antioquia hoy es pensionado, otro hermano fue sociólogo y trabajó en la Normal de La Cruz.

No tuve la oportunidad de salir a prepararme como mis hermanos; primero porque no permitieron que saliera a trabajar lejos y segundo porque no habían establecimientos educativos cercanos, las vías de comunicación malas y lejanas; entonces como estaba lista para la docencia fui profesora durante ocho años en lo rural y treinta (30) años en lo urbano o sea en La Cruz.

En ese bonito tiempo, los nombramientos los hacía el Supervisor Escolar, de acuerdo a la petición del padre y madre de la que iba a ser nombrada, los llevaba a Secretaría de Educación donde les daban el visto bueno.

El salario lo pagaba el departamento y en cada municipio nos pagaba el agente de Rentas, tocaba de comprar un formato que decía nómina de empleados, se llenaba con el nombre del lugar donde estaba trabajando, nombre de la escuela, días que había trabajado en números y letras, el salario lo firmaba el inspector local, el inspector escolar con su sello. El salario fue de $\$ 70$ pesos, $\$ 5$ para alimentación y $\$ 2$ pesos la pieza.

Fui egresada en el mes de julio de 1952, de la escuela Rural de señoritas de la Cruz del Mayo, dirigida por la señorita Nohemí Salcedo de la ciudad de Pasto, el tiempo de estudio fue de 3 años, las materias que se estudiaban eran matemáticas, geometría, castellano y caligrafía, religión e historia sagrada, geografía, historia Patria, cívica, ciencias naturales, educación física, pedagogía, agricultura, trabajos manuales, culinaria, dibujo, música y canto, urbanidad, biblioteca, práctica pedagógica en la escuela.

El primer año nos mandaban a observar clases durante un día en un curso; esto era cada mes. El segundo año, íbamos a dictar clase un día en la semana en diferentes cursos. En el tercer año, preparábamos clase para una semana mensual en el curso que nos asignaran. Las clases tenían tres puntos: Introducción, medio de clase y fin de clase. 
Introducción. Se hacía con un canto, con un cuento o una charla, duración o tiempo 5 minutos.

Medio de clase. Presentación y observación del material que íbamos a utilizar en clase, para entrar al desarrollo del tema, tiempo 35 minutos.

Fin de clase, en un tiempo de 5 minutos, por medio de preguntas, el maestro, se daba cuenta si hubo asimilación del tema. La jornada laboral era de 8 horas diarias: de 7 a 12 de la mañana y de 1 a 5 de la tarde de lunes a sábado. Los materiales que se utilizaban tablero, tiza, paisajes, carteleras, objetos del medio, siluetas, colores, mesa de arena, libro Alegría de leer.

\section{Resultados}

Metodología. Los primeros años enseñábamos el método de palabras normales, con el que aprendimos en la escuela anexa a la normal. La escuela tradicional no tenía curso de jardín, ni kinder, ni transición, o sea preescolar, para matricular un alumno o alumna a primer año de primaria, el requisito importante era haber cumplido 7 años.

Después de enseñar las vocales, se enseñaba la primera palabra normal mamá. La palabra de la escritura mamá era en dos tipos de letra: manuscrita e imprenta.

\section{mamá letra manuscrita \\ mamá letra imprenta.}

Después de aprender la palabra mamá, se la descomponía en silabas, para llegar al sonideo de la consonante. Conocido el sonideo de la consonante, se la acompañaba con las vocales así:

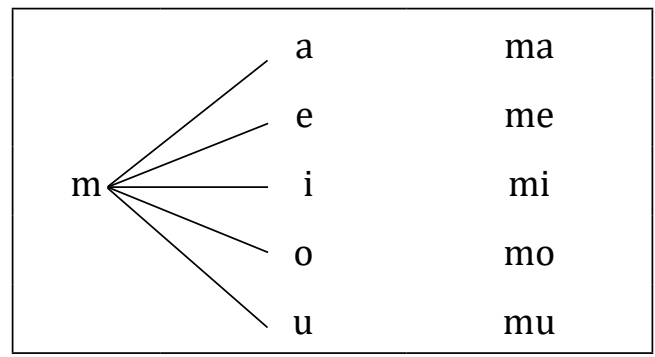

Con estas palabras se enseñaba las palabras

$$
\text { memo - mema - mami - amo - ama }
$$

Con las palabras anteriores se enseñaba frases:

mi mamá me ama

mi mamá me ama

amo a mi mamá

amo a mi mamá 
Antes de enseñar a leer y escribir había un mes para adiestramiento de la mano; amasando arcilla hacen bolitas, rosquitas, ollas, cazuelas, en el cuaderno planas de palitos parados, acostados, inclinados; planas de gusanitos, culebras, curvas, colombinas, cruces, el signo $\mathrm{X}$, palitos que suben y bajan, etc.

Para enseñar con el método global, los alumnos de primero tenían que saber las vocales, con el método global después de la introducción en la clase, se presentaba la frase que se quería enseñar, por ejemplo.

$$
\text { mi mamá me ama (letra script) }
$$

Aquí ya no aparece letra, manuscrita, ni letra imprenta sino la letra script, no se le daba el sonido de la $m$ al niño, el objetivo era que el niño aprenda a leer y escribir de memoria las frases que se enseñaban.

Para mí este método global no lo acepté porque el niño memorizaba demasiado y no sabía el significado de las palabras, ni de las frases, para los padres de familia era preocupante porque no se veía en los primeros meses del año que el niño tuviera buen rendimiento en letra y escritura.

El método que me hizo maestra de verdad fue el de palabras normales, los niños a los tres meses leían y escribían con consonantes que se habían enseñado, los padres de familia llegaban a felicitar y al terminar el año leían periódico, revistas, afiches, no perdían el año aprovechaban el primer año para seguir con su segundo año.

Para evaluar, se formulaban preguntas, se utilizaba el tablero, cartoncitos con las letras, también con números, con dibujos, con palabras. Al final del año se hacían exámenes orales en un día especial, ante un jurado de 5 personas escogidas por el director o directora (Alcalde, inspector de Policía, personas importantes de la ciudad, padres de familia). La educación de esa época era rigurosa, exigente, memorística; el niño no intervenía en clase, tenía la palabra el maestro, había mucho respeto, los niños que no cumplían con su deber eran castigados con correa, con regla, con regaños de voz fuerte.

La educación era muy exigente, los padres de familia empezaban con la exigencia, mi mamá me levantaba muy temprano a las dos de la mañana y me colocaba a ser roscones y a las seis me mandaba a bañarme y luego tenía que irme a la normal, antes de las siete teníamos que estar en clase trabajando, presentando lecciones para cada día a la señorita Ernestina Bolaños y el padre Luis Alberto Puertas, todos los maestros eran exigentes y decían vamos a revisar tareas y los padres en ese tiempo no se preocupaban como ahora, y solo el niño asumía la responsabilidad.

Las clases eran muy exigentes nos daban teoría y práctica, y cuando era teoría tocaba de estar en el aula. El padre de familia no se dedicaba a los hijos y solo se buscaba acabar el grado quinto. El ser maestra me impulsó porque en ese tiempo no había otra profesión y me gustó iniciar este oficio, en mi grupo éramos treinta mujeres en donde nos formaron muy bien con muchos valores, y el nombramiento lo entregaba el supervisor escolar donde había la necesidad dentro de la región. De mi experiencia como maestra lo más grato fue que los niños salieran bien preparados, tengo estudiantes en el Senado, en 
la Cámara, en Estados Unidos, en Europa, en todo el país. Y muchos vienen a visitarme y me agradecen por la labor cumplida. De mis compañeras de grupo eran de muchos sitios del país, pero de La Cruz está Aleja Rebolledo y Carmen Muñoz. La práctica era muy exigente y nos entregaban los temas y éramos muy cumplidas con mucho ánimo y no había preferencia y todas nos dedicábamos a preguntar, consultar, todo lo referente a cada tema y al final nos evaluaban la práctica para graduarnos.

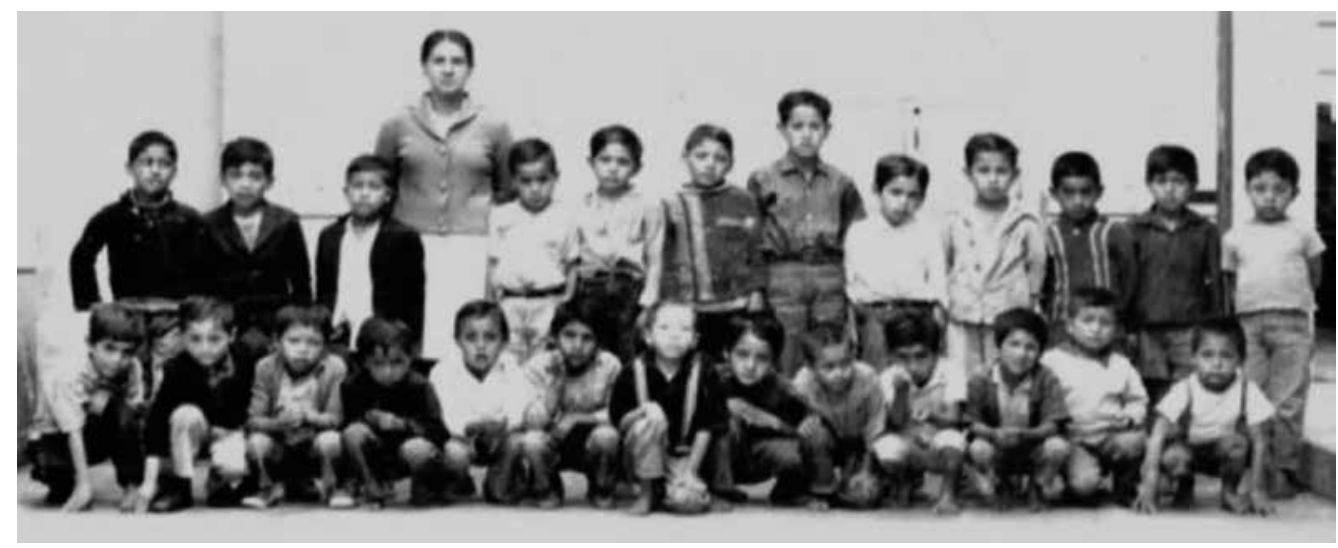

Fuente Ida Victoria Bolaños, 1965.

La cívica y la urbanidad nos enseñaban primero como se debía comportar en el hogar, como debía ser el manejo en la calle saludar, sonreír, ceder el andén, como se manejaba en un paseo, en una casa de visita, que se hacía como se atendía a la gente, la urbanidad era bien detallada era el manejo en la casa del vecino, el comportamiento en casa y fuera de la casa, en la escuela, en un viaje, no rayar las paredes y como se maneja en los desfiles, en las procesiones y siempre nos estaban vigilando.

En tres años nos preparaban para ser maestras y aprendimos mucho como íbamos a trabajar y enseñar, la experiencia siempre forma.

El mensaje para los maestros de este tiempo, que tengan amor al estudio y mirar la situación económica de sus padres hasta donde son capaces, ser cumplidos en su deber y ayudarse unos a otros"18 (Bolaños Ida, 2016).

\section{Discusión}

El trabajo de las maestras rurales siempre estuvo enfocado en la entrega y dedicación para formar los niños y jóvenes que pudieran demostrar sus capacidades con el único propósito de salir adelante y superar las múltiples dificultades que se presentan en el transcurso de su diario vivir. El trabajo de las

18. Entrevista a Bolaños Cerón, Ida Victoria, maestra rural, La Cruz Nariño, 2017. 
maestras se contempla en la rigurosidad de su entrega para formar y preparar jóvenes de bien, con valores para enfrentar los retos de la sociedad.

La capacidad de entrega de estas maestras, estaba dada en la disponibilidad que le ofrecían a las comunidades ya que en las mañanas trabajan con los niños y en las tardes ofrecían sus servicios a los adultos donde se les preparaba en lectura y escritura y en las noches se trabajaba con las mujeres tejido, bordados, costura para apoyar que las mujeres tengan ratos de descanso en otras actividades propias del momento y de la época.

El trabajo de formación y preparación dentro de las normales era de entrega y vocación para fundamentarse muy bien y luego toda esa orientación debía de tener un conocimiento donde debía ser aplicado a sus niños y jóvenes quienes eran instruidos en las áreas básicas y con ello fortalecer su enseñanza-aprendizaje.

La escuela con un solo maestro conducía a una visión de la institución escolar centrada en el auto aprendizaje y desarrollo individual del estudiante (una nueva concepción del proceso enseñanza-aprendizaje) o basada en el desarrollo social (escuela como centro de la comunidad). En el marco de una formación ciudadana y humanista, necesaria para una vida digna y con la finalidad de formar desde la escuela, al sujeto rural como constructor y actor de su propio desarrollo.

La evaluación dada a las maestras rurales es muy gratificante ya que entregaron toda la disponibilidad posible para contribuir con la formación de los niños y niñas de cada comunidad y contexto, logrando avances tanto en conocimientos, habilidades, valores, actitudes y visiones de la enseñanza y el aprendizaje que fortalecieron las bases de la sociedad empeñada en el desafío de salir del retraso y buscar las metas de la superación y el cambio personal, que determinen que la educación apunta a las nuevas rutas que enfocan cambios estructurales en el saber y hacer.

Las maestras rurales, fueron mujeres guerreras de botas y sudaderas que entregaron su vida para contribuir con el mejoramiento del país, llevan en su memoria los juegos, carcajadas, gritos, sonrisas, cuentos y nostalgias de niños y niñas que ya crecieron y que son la esencia viva de su trajinar por las diferentes carreras que ha trazado su destino y que florecen en sus expectativas que construyen en su mundo social. Maestra Rural de carácter y convicción.

Al comparar las maestras rurales y las maestras de hoy, se detecta que estas maestras entregaban su vida a la formación de niños y niñas en el país con horarios de día y de noche sin importar medir el tiempo, trabajaban con las uñas sin esperar el apoyo del gobierno en la dotación de materiales (tiza, tablero y lengua) y los niños son preparados en valores y normas de urbanidad; el trabajo de hoy es diferente se cuenta con materiales didácticos y tecnológicos que hacen que la educación sea más amena y los niños y padres de familia están comprometidos con la orientación que se recibe y los horarios son establecidos estrictamente sin regalar nada a cambio y el resultado son niños más rebeldes, altaneros, groseros y menos comprometidos con sus labores académicas. 


\section{Conclusiones}

La reforma constitucional de 1936 consagró la libertad de enseñanza y creó las Escuelas Normales Rurales, con el único propósito de formar nuevas maestras con fundamentos en valores y en formación epistemológica, las cuales ingresarían a la fuerza laboral para mejorar el alto índice de analfabetismo que había en cada región y que afectaba el desarrollo socio económico del país.

El papel desempeñado por las normales rurales en cada departamento, municipio y región fue contribuir a la búsqueda del camino, hacia la prosperidad donde la escuela anexa era un laboratorio de pedagogía práctica con plena autonomía en el intelecto centrada en la formación del alumno y el profesor era un guía que acompañada los fundamentos de la práctica para formar la nueva generación de maestras que apoyarían el desarrollo industrial y cultural de Colombia.

El punto neurálgico y decisivo, en la formación de los maestros en las Escuelas Normales Rurales era preparar a la población para la fuerza laboral del trabajo, para la formación cultural y en valores, desarrollando procesos de industrialización y urbanización, creando la necesidad de una mano de obra calificada y el surgimiento de la alfabetización como condición de la vida.

Las Normales rurales, permitieron la formación de una nueva cultura y desarrollo de un nuevo hombre estructurado en la formación de un intelectual formador de generaciones comprometido con el desarrollo agrícola, financiero, comercial y laboral, donde se sientan las bases de las prácticas pedagógicas, de los modelos y del quehacer pedagógico con el único propósito de formar a sus maestras en conocimientos y valores marcados en el progreso de las comunidades.

La maestra rural jugó un papel importante en el desarrollo intelectual del país, ya que fueron formadas en varias áreas del conocimiento y además reforzaban las artes, tejidos, bordados, culinaria, capaz de transformar la cultura de los pueblos y lograr formar maestros para la educación primaria y secundaria a partir de las nuevas corrientes pedagógicas. 


\section{REFERENCIAS BIBLIOGRÁFICAS}

Betancourt Mejía, G. (Compilador). Documentos para la historia del planteamiento integral de la educación. Informe para el proyecto del Primer Plan Quinquenal de educación (Colombia 1956). Vol. II. Bogotá: Universidad Pedagógica Nacional, 1984.

Bolaños Cerón, Ida Victoria. Maestra Rural de señoritas de La Cruz, Nariño, 2017.

Decreto 356 de 27 de agosto de 1874. Art. 1. Presidente Santiago Pérez, Escuelas Normales de mujeres. Estados Unidos de Colombia.

El Tiempo, 22 de noviembre de 1935. Memoria de 1938 (cuadro en anexo) señala sin embargo, 14 maestros y 11 maestras ambulantes este año (1935), primera edición. Número 1531.

González, Fernán. "Educación y estado en la historia de Colombia”. Revista Colombiana de Educación, No. 4 (1979): 81.

Helg, Aline, La educación en Colombia: 1918-1957, Bogotá: Universidad Pedagógica y Tecnológica de Colombia. Plaza y Janés, 1987.

López de Mesa, Luis. “Tres instituciones pedagógicas Nacionales”. Revista de Indias. Vol. 1, No. 7, Bogotá (1937): 54.

Memoria del Ministro de Educación, 1936.

MEN, Memoria de 1935, 250-251.

Ministerio de Educación Nacional. Plan de estudios para las Escuelas Normales Regulares. Bogotá: Imprenta Nacional, 1946.

Palacios Arcos, Irma Cecilia. Maestra Rural de señoritas de la Cruz Nariño, 1953.

Ramírez, M. T. y Pachón, Á. La infraestructura de transporte en Colombia durante el siglo XIX. Bogotá: Banco de la República y Fondo de Cultura Económica, 2006.

República de Colombia - Memoria de 1935. 31

República de Colombia. "Memoria del Ministerio de Educación al congreso de 1946". Bogotá: Imprenta Nacional, 1936, 13.

Sáenz, J.; Saldarriaga, O. y Ospina, A. Mirar la infancia: pedagogía, moral y modernidad en Colombia, 19031946. Medellín: Editorial Universidad de Antioquia, 1997.

Triana Ramírez, Alba Nidia. "Escuelas Normal, Rural, Agropecuaria y de Campesinas en Colombia: 1934 1974", Revista Historia de la educación en Colombia, Vol. 13, No. 13 (2010): 205. 